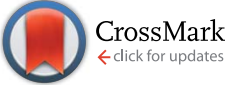

Cite this: RSC Adv., 2014, 4, 45696

Received 8th August 2014

Accepted 8th September 2014

DOI: $10.1039 / c 4 r a 08361 j$

www.rsc.org/advances

\section{Fabrication of biocompatible free-standing nanopatterned films for primary neuronal cultures $\uparrow$}

\author{
F. Cesca,,$t^{\mathrm{a}}$ T. Limongi, $\hat{t}^{\mathrm{b}}$ A. Accardo, ${ }^{\mathrm{c}}$ A. Rocchi, ${ }^{a}$ M. Orlando, ${ }^{a}$ V. Shalabaeva, ${ }^{c}$ E. Di \\ Fabriziot $^{* b d}$ and F. Benfenatita
}

Devising and constructing biocompatible devices for nervous system regeneration is an extremely challenging task. Besides tackling the issue of biocompatibility, biomaterials for neuroscience applications should mimic the complex environment of the extracellular matrix, which in vivo provides neurons with a series of cues and signals to guide cells towards their appropriate targets. In this work, a novel nanopatterned biocompatible poly- $\varepsilon$-caprolactone (PCL) film is realized to assist the attachment and growth of primary hippocampal neurons. Costly and time-consuming processes can be avoided using plasma-surface nanotexturing obtained by a mixed gas $\mathrm{SF}_{6} / \mathrm{Ar}$ at $-5^{\circ} \mathrm{C}$. The intrinsic composition and line topography of nanopatterned PCL ensure healthy development of the neuronal network, as shown by confocal microscopy, by analysing the expression of a range of neuronal markers typical of mature cultures, as well as by scanning electron microscopy. In addition, we show that surface nanopatterning improves differentiation of neurons compared to flat PCL films, while no neural growth was observed on either flat or nanopatterned substrates in the absence of a poly-D-lysine coating. Thus, we successfully optimized a nanofabrication protocol to obtain nanostructured PCL layers endowed with several mechanical and structural characteristics that make them a promising, versatile tool for future tissue engineering studies aimed at neural tissue regeneration.

\section{Introduction}

The engineering of biocompatible devices for biomedical applications represents one of the main challenges faced by the fields of bioengineering and materials science. While this is true for all the aspects of regenerative medicine, biocompatibility becomes an essential issue when trying to devise novel strategies for neuroscience applications. In fact, neurons are extremely demanding, especially for what concerns their ability to adhere, grow and differentiate on specific substrates. In general, surface roughness and nano-scale topography are known to affect the ability of neural cells to grow and differentiate. ${ }^{1,2}$ Substrate topography, especially at the nanoscale, is considered a promising tool for the manipulation of cell

${ }^{a}$ Neuroscience and Brain Technologies Department, Istituto Italiano di Tecnologia, Via Morego 30, 16163 Genova, Italy

${ }^{b}$ King Abdullah University of Science and Technology, PSE and BESE Divisions, Thuwal, 23955-6900, Kingdom of Saudi Arabia

'Nanostructures Department, Istituto Italiano di Tecnologia, Via Morego 30, 16163 Genova, Italy

${ }^{d}$ BIONEM, Bio-Nanotechnology and Engineering for Medicine, Department of Experimental and Clinical Medicine, University of Magna Graecia Viale Europa, Germaneto, 88100 Catanzaro, Italy

$\dagger$ Electronic supplementary information (ESI) available. See DOI: 10.1039/c4ra08361j

\$ Equal contribution. function and the guidance of tissue regeneration. Thus, engineering of biomaterials has become increasingly more focused on structuring surfaces at the micro- to nano-scale, to optimize cell adhesion and protein adsorption, which is crucial to achieve optimal implant performance.

In general, biomaterials for tissue engineering applications mimic the structural complexity of the extracellular matrix (ECM). ${ }^{3}$ The rigidity of ECM proteins such as fibronectin, vitronectin and laminin endows the substrate with the appropriate stiffness to counteract the contractile forces imposed by the cells. Cells adhere to the substrate by establishing specialized adhesive sites, named focal contacts, where membrane receptors are simultaneously linked to extracellular ligands and to the intracellular cytoskeleton. ${ }^{4}$ ECM proteins play a fundamental role in several aspects of neuronal physiology. They direct axon pathfinding and neuronal migration of developing neurons by attracting or repelling axonal growth cones until they reach their final target. Under pathological conditions, such as nerve injury or neuropathies, the same molecules are able to drive axonal regeneration, eventually leading to a partial functional recovery. ${ }^{5,6}$ Synthetic interactions may thus be engineered to serve as a potent signalling mechanism for use in regenerative medicine. Recent developments in photolithography and microfabrication allow producing substrates with micro- and nano-scale features in the range of the native topography of ECM molecules. ECM components often contain 
fibrous structural elements that can be recapitulated in $2 \mathrm{D}$ systems through ridge-groove nanograting features. Thus, microfabrication strategies hold a great potential to reconstruct native nano-topography both in vitro and in vivo. Photolithography and soft lithography are the most common microfabrication techniques for the production of reproducible nanofeatures in a variety of soft materials. Since these techniques are usually expensive and time consuming, various nanofabrication methods have been developed, such as electrospinning, chemical etching, polymer demixing, and colloidal lithography. ${ }^{7,8}$ In particular, plasma-surface modifications are gaining popularity in the biomedical field, as they represent an extremely promising, economical and effective material processing technique. This technology allows changing several properties of the materials, such as chemical composition, wettability, chemical inertness, metal adhesion, refractive index, hardness and biocompatibility. ${ }^{9-11}$

Among the currently employed materials in neuroregeneration research, an important role is played by poly- $\varepsilon^{-}$ caprolactone (PCL), a well known biodegradable and biocompatible polyester that has been used in a variety of applications ranging from in situ drug delivery ${ }^{\mathbf{1 2}}$ to cell migration, proliferation ${ }^{13}$ and bio-scaffolds manufacturing. ${ }^{14}$ Among other techniques, PCL was used in the form of electrospun nanofibers, combined with other collagens by solvent casting, for the preparation of porous scaffolds. ${ }^{15}$ Moreover, it has been extensively used to produce suitable platforms for neuronal cell growth and proliferation. ${ }^{16}$ In the present work we describe a novel fast-prototyping method involving a single-step plasma treatment that allows the realization of novel PCL biocompatible and bioerodible surfaces to assist the attachment and growth of primary hippocampal neurons.

\section{Results and discussion}

\section{Fabrication of nanopatterned PCL substrates}

To achieve surface nanopatterning, PCL was etched by using a mixed gas $\mathrm{SF}_{6} / \mathrm{Ar}$ at $-5{ }^{\circ} \mathrm{C}$, to prevent any possible partial melting of the PCL film during the plasma process (Fig. 1a). When considering a biocompatible surface, the interaction between the cell and the material is influenced not only by the surface topography, but also by its physicochemical properties including surface wettability and charge. Wettability of biomaterials by liquid media or body fluid is one of the most important properties of a solid surface, which has to be taken into consideration for all in vitro and in vivo cell and tissue engineering applications. To estimate the surface wettability of our nanostructured PCL substrates, we measured the contact angle before (Fig. 1b) and after (Fig. 1c) the plasma treatment, obtaining values that varied from $90.5^{\circ}$ (hydrophobicity/ hydrophilicity threshold; PCL film on the Si wafer after spinning and soft-bake) to $106.8^{\circ}$ (low hydrophobicity; PCL film after plasma roughening). The surface morphology and roughness of our PCL devices were quantitatively characterized by using atomic force microscopy. Fig. 1d and e show the 3D Atomic Force Microscopy (AFM) images of the flat and nanostructured PCL surfaces, respectively. In the case of a just spin-
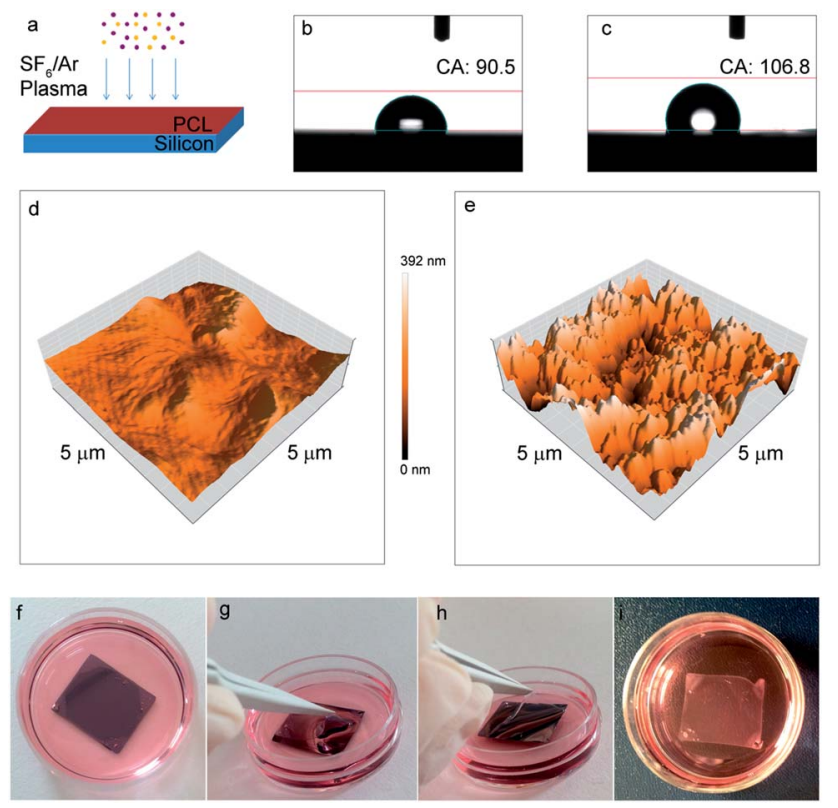

Fig. 1 PCL nanopatterned surface fabrication, characterization and peel-off. (a) Schematic view of the fast single-step plasma etching for the nano-texturing of PCL films. Surface water contact angle (b and $c$ ) and AFM measurements ( $d$ and e) of merely spin-coated (b and d) and nanopatterned ( $c$ and e) surfaces. Picture of the nanopatterned PCL film tightly adhering to the silicon substrate (f); peeling-off procedure ( $g$ and $h$ ); final floating nanostructured film (i).

coated and soft-baked PCL surface (Fig. 1d) we obtained, for a typical $5 \times 5 \mu \mathrm{m}$ area, a $R_{\mathrm{a}}$ value of $18.9 \mathrm{~nm}$ and a RMS of 30.1 $\mathrm{nm}$, whilst for the same area of a nanopatterned PCL surface (Fig. 1e), we measured a $R_{\mathrm{a}}$ of $56.8 \mathrm{~nm}$ and a RMS of $75.2 \mathrm{~nm}$. The Si wafer acts as a support to spin and texture the PCL film, providing also the stability that is required during all the standard cell culture procedures such as sterilization, seeding and medium exchange. The thin PCL film attached on the silicon substrate can be maintained successfully in the cell culture medium for weeks (Fig. 1f). During this period, it is always possible to carry out the peeling-off of the film, whenever its "free-standing" use and/or its transfer onto other substrates is required (Fig. 1f-i).

\section{Biocompatibility of PCL substrates with primary neuronal cultures}

In order to determine whether our nanopatterned devices were compatible with the growth and differentiation of primary neurons, we evaluated the viability of neural cultures grown on flat and nanopatterned PCL films, compared to cultures grown on standard glass supports (Fig. 2). All the substrates were precoated with poly-D-lysine (see Experimental section for details), since no neural growth was observed on either glass or PCL in the absence of coating (data not shown). Interestingly, viability of neural networks grown on nanopatterned substrates was comparable to what observed under standard culture conditions. On the contrary, increased cell death was apparent in cultures grown on flat PCL devices (Fig. 2b). 

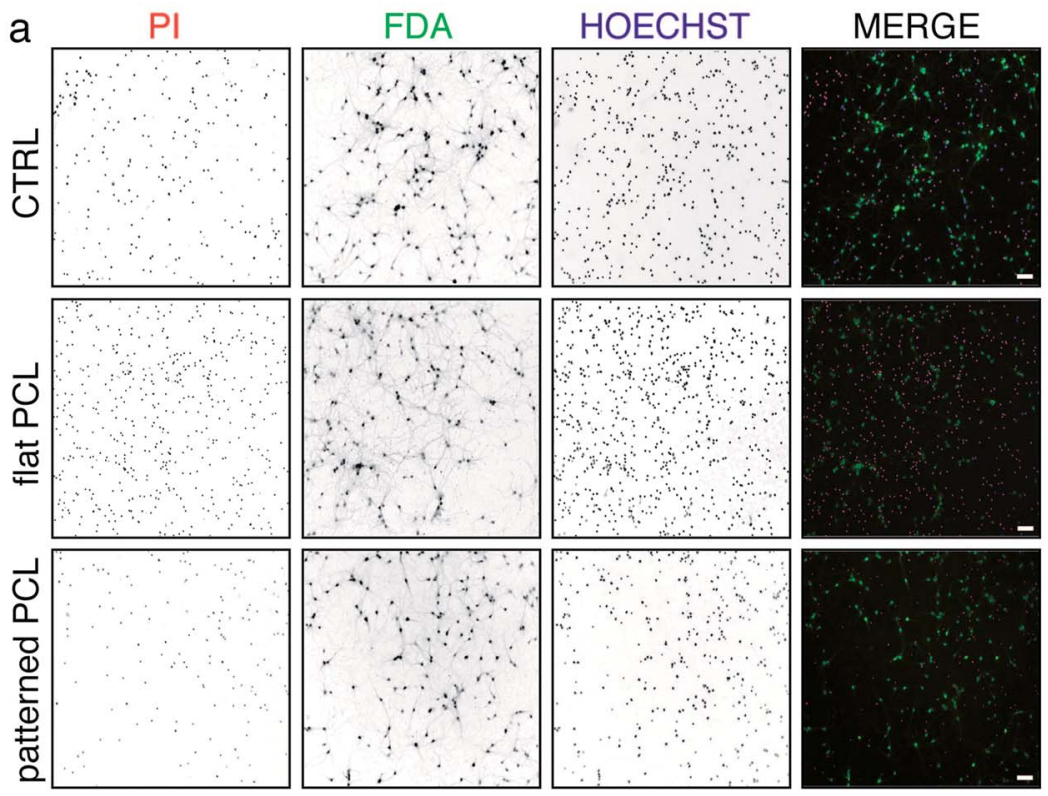

b

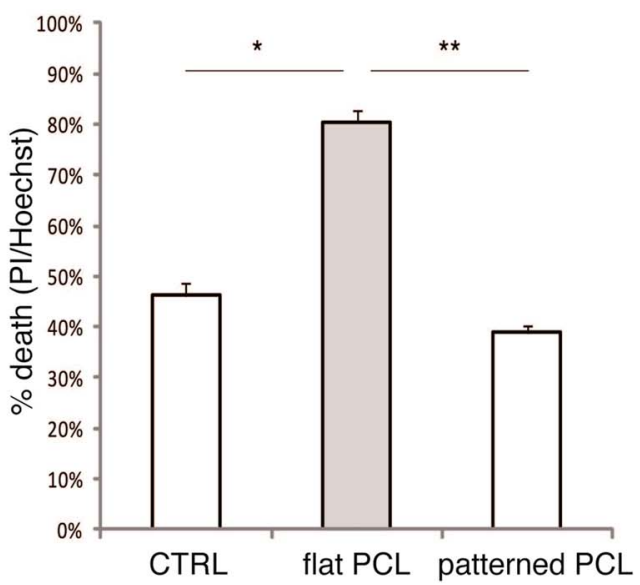

Fig. 2 Biocompatibility of PCL substrates with primary neuron cultures. Hippocampal neurons grown on standard culture dishes (CTRL), flat and patterned PCL substrates for 7 days were incubated in extracellular medium containing PI, FDA and Hoechst (see Experimental section) and immediately imaged. Representative images are shown in panel a (scale bars: $50 \mu \mathrm{m}$ ). Quantitative analysis of neuronal death, shown in panel b, was performed by calculating the percent ratio of PI-positive nuclei on total (Hoechst-positive) nuclei (PI/Hoechst) and expressed as means \pm S.E.M. ( $n=3$ independent preparations). Statistical analysis was performed by one-way ANOVA, followed by the Bonferroni post hoc test (*P< $0.05 ; * * P<0.001)$

With the aim of monitoring the morphology of cultured cells, we plated neurons on poly-D-lysine coated flat and nanopatterned supports, let the cultures grow for 7 days in vitro (DIV), and processed them for scanning electron microscopy (SEM; Fig. 3). In agreement with our viability data, sparse neurons were observed on flat films, which formed a scant network. Neurons grown on nanopatterned supports were instead healthy, as
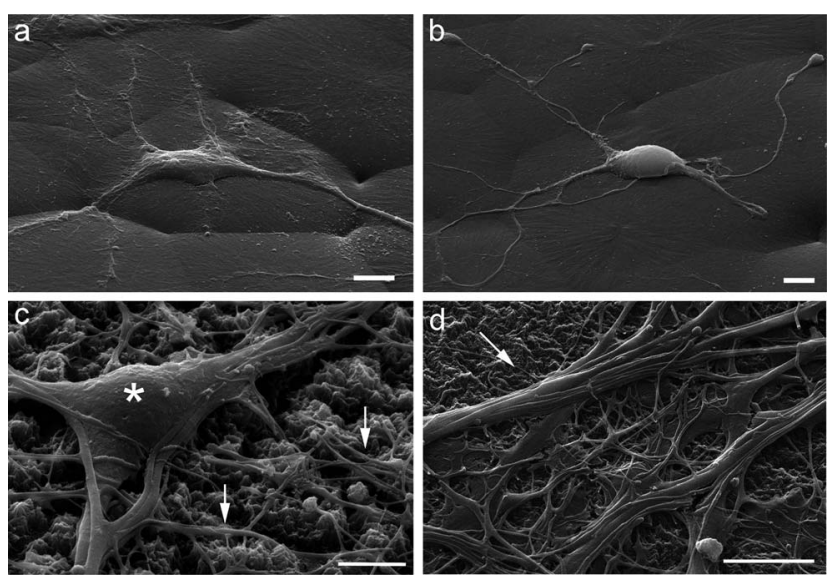

Fig. 3 SEM images of primary hippocampal cultures plated on flat (a and b) and nanopatterned (c and d) PCL substrates. Cells were grown for 7 days, fixed and processed for SEM. Only few cells grew on flat films, forming a sparse network. On nanopatterned substrates, instead, neurons were healthy, as indicated by the smooth surface of cell bodies (asterisk) and by the dense network of neurites (arrows), which grew in tight adhesion with the substrate. Scale bars: $5 \mu \mathrm{m}$. indicated by the smooth cell bodies, and formed a well-developed network in close contact with the substrate.

To analyze in more detail the development of neuronal networks grown on flat and nanopatterned PCL substrates, we processed some samples for confocal microscopy analyzing a range of markers typically expressed in mature neuronal cultures (Fig. 4). To visualize synaptic connections, we analyzed the expression and distribution of the synaptic vesicle protein synapsin I (syn I, Fig. 4a and a'); to characterize the adhesion properties to the substrate, we used antibodies to the neural cell adhesion molecule (NCAM, Fig. $4 \mathrm{~b}$ and $\mathrm{b}^{\prime}$ ); to get more insights into the differentiation and functionality of the cells, we monitored the axonal marker SMI-31 (Fig. $4 \mathrm{c}$ and $\mathrm{c}^{\prime}$ ) and the distribution of voltagedependent $\mathrm{Na}^{+}$channels $\left(\mathrm{Na}_{v}\right.$, Fig. $4 \mathrm{~d}$ and $\left.\mathrm{d}^{\prime}\right)$. Neurons grown onto nanopatterned PCL supports were able to form a rich axonal/dendritic network that exhibited a dense pattern of synaptic contacts (Fig. $4 \mathrm{a}, \mathrm{a}^{\prime}$ and $4 \mathrm{c}, \mathrm{c}^{\prime}$ ). In addition, $\mathrm{Na}^{+}$ channels localization was restricted to the axonal initial segment, as expected (Fig. $4 \mathrm{~d}$ and $\mathrm{d}^{\prime}$ ), thus indicating that these cultures expressed all the components required for proper excitability and connectivity. Of particular interest was the pattern of NCAM immunoreactivity (Fig. $4 \mathrm{~b}$ and $\mathrm{b}^{\prime}$ ) that was very highly expressed in a dense and ramified network all around the cell bodies and neurites of neurons, testifying the very strong adhesion of the cells to the substrates. Neurons grown on flat PCL supports displayed a similar pattern of staining, however being the network less dense, they were characterized by more sparse synaptic 

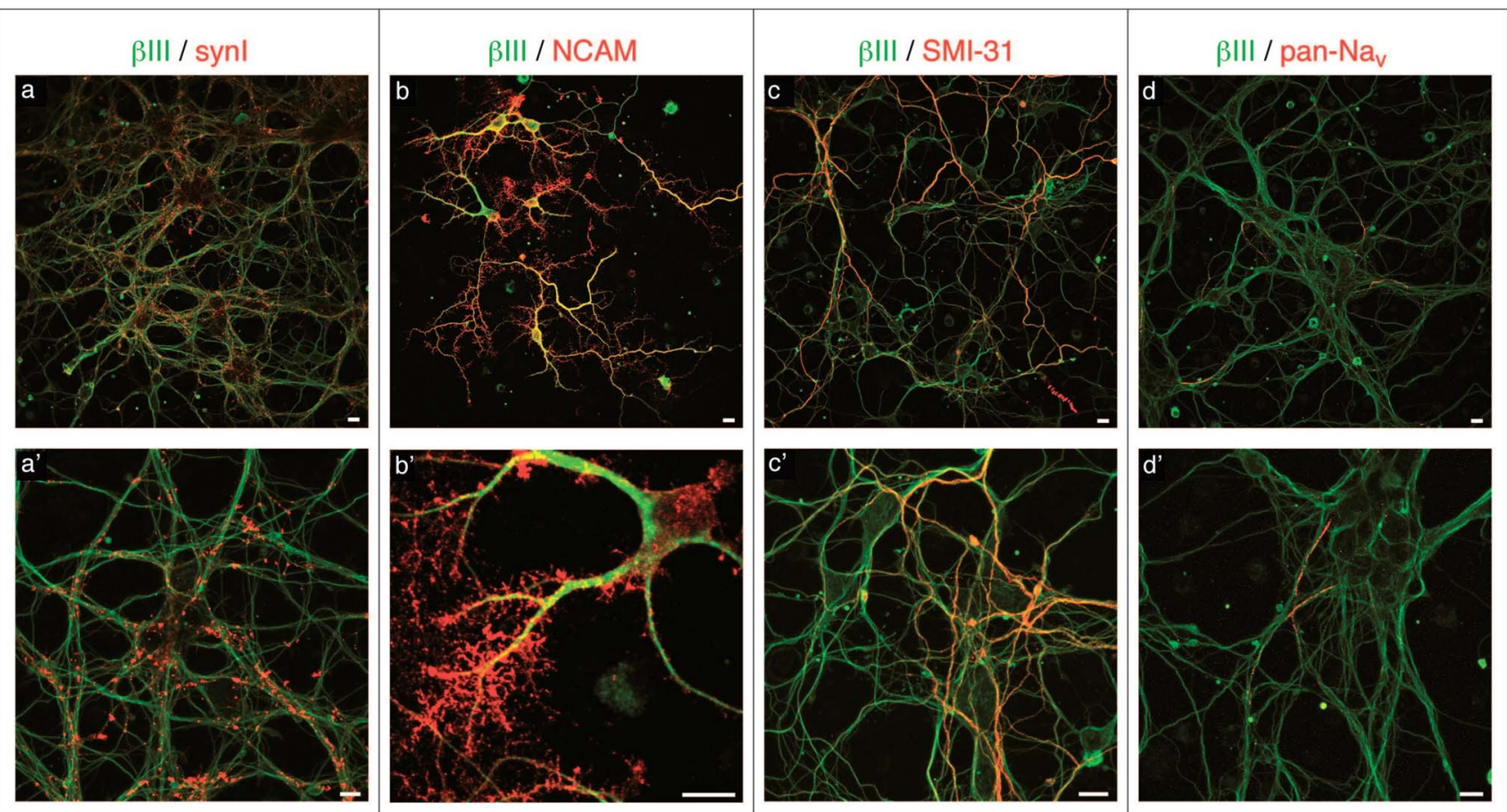

Fig. 4 Confocal images of primary hippocampal cultures plated on nanopatterned PCL substrates at two magnifications (upper and lower rows). Neurons were grown for 7 days on the substrates, fixed and processed for confocal microscopy. A range of markers typically expressed in mature neuronal cultures were analysed, as indicated: $\beta$ III, neuronal class III $\beta$-tubulin (green channel); synl, synapsin I; NCAM, neural cell adhesion molecule; SMI-31, phosphorylated neurofilament heavy chain; pan- $\mathrm{Na}_{v}$. pan- $\mathrm{Na}^{+}$channels (red channel). Neurons formed a rich network, characterized by a dense pattern of synaptic contacts. $\mathrm{Na}_{v}$ localization was restricted to the axonal initial segment. NCAM-immunoreactivity $\left(b\right.$ and $b^{\prime}$ ) was highly expressed all around the cell bodies and neurites, indicating a very strong adhesion of the cells to the substrates. Scale bars: $10 \mu \mathrm{m}$

contacts and a less prominent expression of adhesion molecules (ESI Fig. S1†).

\section{Nanopatterned PCL substrates promote neuronal growth}

All together, our viability, electron microscopy and confocal microscopy data suggest that surface nanopatterning promotes the formation of healthy neural networks, possibly better than flat films. To provide a quantitative readout of these observations, we evaluated neurite outgrowth on the various substrates by performing Sholl analysis, which is a widely accepted method to evaluate the complexity of neural networks (see, for example ref. 17). As shown in Fig. 5a, neurons grown on nanopatterned PCL substrates displayed more complex arborization compared to cells grown on flat films, thus indicating that surface patterning could indeed promote neural adhesion and differentiation to levels comparable to what is normally achieved under standard culture conditions (CTRL; Fig. 5b).

Our results thus emphasize the importance of process optimization for the realization of biocompatible, free-standing films of polymer endowed with flexibility, strength and ability to adhere to different substrates. This system of nanofabrication allowed us to investigate the effects of nanotopography on neuronal cell growth providing the manufacture of an inexpensive, reproducible and scalable pattern. Such system consists of a fast-prototyping method involving a single- step physical etch performed by plasma nano-roughening without any further chemical modification, thus consistently reducing the time required to obtain a ready-to-use substrate. The ultrathin PCL films obtained by this method present a nanoporous air surface and exhibit flexibility and sufficient mechanical strength for sterilization and clinical handling. The nano-featured surfaces are attractive for neuronal cells because they mimic both the mechanical and wettability properties of the extracellular matrix. The study demonstrates that, in the absence of other biochemical cues, the intrinsic composition and line topography of nanopatterned PCL is sufficient to support growth of primary neurons. Nanoscale features, introduced on the polymer surface during plasma treatment, favour cell attachment on more hydrophobic surfaces as previously demonstrated by our group for super-hydrophobic devices. ${ }^{2}$ One appealing application for the nanopatterned supports described in this work is the possibility to achieve directional neuronal growth, thus possibly guiding neurites during regeneration processes in vivo, similar to what was obtained by aligned chitosan-PCL fibers. ${ }^{18}$ We are implementing effective and reproducible methods to produce spatially controlled nanotopography using stencil masks for the selective plasma etch. Changing the topographic properties of surfaces, areas differentially permissive to the adhesion of neuronal cell bodies and processes will be realized on the biocompatible devices. In perspective, it will be interesting to modulate neural growth and 


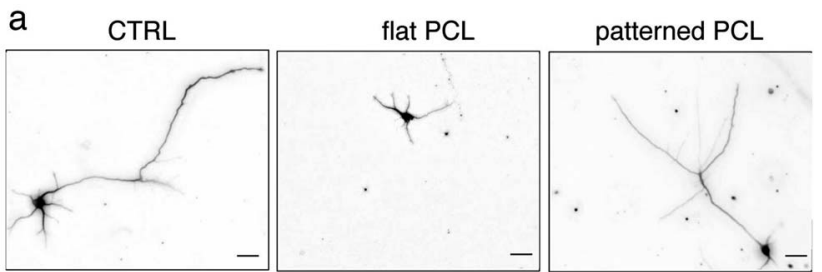

b

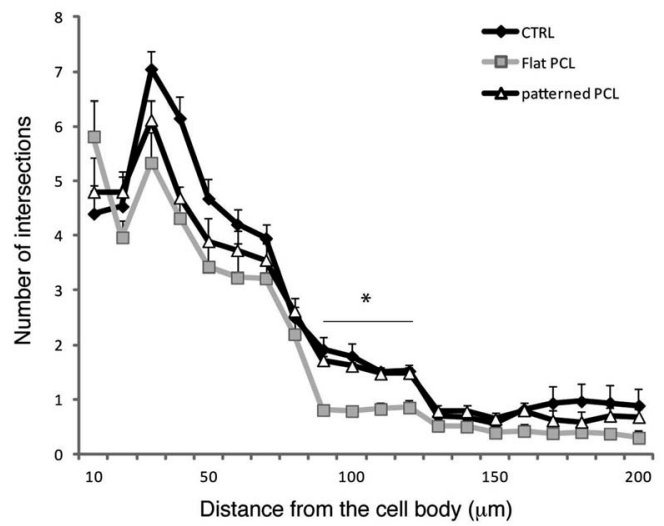

Fig. 5 Nanopatterned PCL substrates improve neurite outgrowth and dendritic branching compared to flat PCL substrates. Hippocampal neurons were plated, fixed at DIV 4 and stained for $\beta$ III-tubulin. Representative images are shown in a (scale bars: $20 \mu \mathrm{m}$ ), quantitative analysis is provided in (b). Data are expressed as means \pm S.E.M. ( $n=$ 150 cells per condition, from 3 independent preparations). Statistical analysis was performed by one-way ANOVA, followed by the Bonferroni post hoc test $(* P<0.05)$. A statistically significant difference between neurons grown on CTRL and patterned PCL vs. neurons grown on flat PCL was detected between 80 and $120 \mu \mathrm{m}$ from the cell bodies (indicated by the black bar). The asterisk indicates that for all the values in the range between 80 and $120 \mu \mathrm{m}$ there is a statistically significant difference $(P<0.05)$, for both "CTRL vs. flat" and "patterned vs. flat".

adhesion by changing the degree of roughness of the PCL supports by varying the plasma etching time, the chamber pressure and the RF/ICP electric power.

In conclusion, the nanopatterned PCL films described in this work represent a versatile tool for future tissue engineering studies and an ideal material for the fabrication of nerve conduits and patches for neuronal tissue regeneration.

\section{Experimental procedures}

\section{PCL films nanofabrication}

The nanotexturing of poly- $\varepsilon$-caprolactone (PCL, Sigma Aldrich Inc.) substrates was performed by a SenTech Inductively Coupled Plasma Reactive Ion Etch (ICP-RIE) system. A 5\% solution of PCL in tetrahydrofuran (Sigma Aldrich Inc.) was prepared using PCL pellets $\left(M_{\mathrm{w}}=77 \mathrm{kDa}\right)$. The pellets were diluted in tetrahydrofuran at $60{ }^{\circ} \mathrm{C}$ through the use of a magnetic stirrer. Afterwards, $\mathrm{Si}\langle 100\rangle$ wafers (Si-Mat) were cut by a diamond tip in single $2 \times 2 \mathrm{~cm}^{2}$ pieces, washed in acetone (Sigma Aldrich Inc.) to remove any possible dust, rinsed in isopropanol (Sigma Aldrich Inc.) and dried under nitrogen. The
PCL solution was then spin-coated on a Si support at $500 \mathrm{rpm}$ for $70 \mathrm{~s}$, obtaining a film whose thickness, measured by a Veeko Dektak profilometer, was $20 \mu \mathrm{m}$. The obtained sample was soft baked at $65^{\circ} \mathrm{C}$ for $5 \mathrm{~min}$ and cooled down at room temperature (RT). Finally, a plasma roughening was performed; the etching was obtained using a mixed gas $\mathrm{SF}_{6} / \mathrm{Ar}(100 \mathrm{sccm} / 10 \mathrm{sccm})$ with an internal chamber pressure of $5 \mathrm{~Pa}$. The RF power and the ICP source were set, respectively, to $30 \mathrm{~W}$ and $800 \mathrm{~W}$. The temperature was kept at $-5{ }^{\circ} \mathrm{C}$ for the whole duration of the process $(90$ s) to prevent any possible partial melting of the PCL film during the plasma process. The AFM analysis on PCL nanostructured surfaces was performed in air by a nanosurf atomic force microscope in tapping mode with a single-crystal silicon tip (nominal radius of curvature $5-10 \mathrm{~nm}$; cantilever vibration frequency $\sim 200 \mathrm{kHz}$ ). Scan areas were $5 \times 5 \mu \mathrm{m}$ with scan rates of $1 \mathrm{~Hz}$. For the analysis of the average roughness $\left(R_{\mathrm{a}}\right)$ and of the mean square roughness (RMS) of the scanned surface profile, the Gwyddion open source software was used. The contact angle was estimated with a KRUSS DSA100 setup by sessile drop method using the Young-Laplace fit.

\section{Substrate preparation for cell seeding}

Single nanopatterned substrates $\left(2 \mathrm{~cm}^{2}\right)$ were placed in $35 \mathrm{~mm}$ tissue culture dishes, sterilized by immersion in ethanol (Fluka, ethanol 02483; $\geq 99.9 \%$ ), washed twice in $\mathrm{H}_{2} \mathrm{O}$, dried in a laminar flow hood and further sterilized by UV irradiation for 90 min. To provide the best biomimetic surface ionic charge condition, scaffolds were immersed in Neurobasal containing 2\% B27 supplement, $2 \mathrm{mM}$ glutamine and antibiotics (maintenance medium) equilibrated with $5 \% \mathrm{CO}_{2}, 95 \%$ humidity for at least $48 \mathrm{~h}$ at $37{ }^{\circ} \mathrm{C}$. After removal of the maintenance medium, substrates were coated overnight with poly-D-lysine (PDL, Sigma Aldrich Inc.) diluted in sterile $\mathrm{H}_{2} \mathrm{O}$ to a final concentration of $0.1 \mathrm{mg} \mathrm{mL}{ }^{-1}$. Before cell plating, PDL was removed and substrates were washed in sterile $\mathrm{H}_{2} \mathrm{O}$ and let dry.

\section{Hippocampal cultures}

Whole brains were extracted from C57B/L6 mouse embryos at day 18 (E18). All experiments were carried out in accordance with the guidelines established by the European Community Council (Directive 2010/63/EU of September 22nd, 2010) and approved by the Italian Ministry of Health. Pregnant females were deeply anesthetized with $\mathrm{CO}_{2}$, embryos were removed and brains were placed in cold Hank's Balanced Salts solution (HBSS). After removal of the meninges the hippocampus was carefully dissected, incubated with $0.125 \%$ trypsin for $15 \mathrm{~min}$ at $37^{\circ} \mathrm{C}$ and mechanically dissociated. Neurons were plated on the PDL-coated nanopatterned substrates in Neurobasal containing $10 \%$ horse serum, $2 \mathrm{mM}$ glutamine and antibiotics (plating medium). 100000 cells were plated on each device, in $200 \mu \mathrm{l}$ of plating medium. After $4 \mathrm{~h}$, the plating medium was removed and replaced with maintenance medium. All culture media and reagents were from Invitrogen (Milano, Italy). 


\section{Viability assay}

The viability of neural cultures on the various substrates was evaluated as previously described. ${ }^{19}$ Briefly, cells were incubated for $3 \mathrm{~min}$ at RT in extracellular medium (EM) ( NaCl $135 \mathrm{mM}$, $\mathrm{KCl} 5.4 \mathrm{mM}, \mathrm{MgCl}_{2} 1 \mathrm{mM}, \mathrm{CaCl}_{2} 1.8 \mathrm{mM}$, glucose $10 \mathrm{mM}$, Hepes $5 \mathrm{mM}, \mathrm{pH}$ 7.4) containing $15 \mu \mathrm{g} \mathrm{mL} \mathrm{m}^{-1}$ fluoresceine diacetate (FDA), $5 \mathrm{mg} \mathrm{mL}{ }^{-1}$ propidium iodide (PI), and $3.3 \mu \mathrm{g} \mathrm{mL}$ Hoechst-33342. After incubation, cells were washed once in EM and immediately imaged. The hardware configuration for the imaging experiments was based on a Nikon Eclipse 80i upright microscope (Nikon Instruments, Prato Calenzano, Italy) equipped with an epifluorescence attachment and a digital camera Nikon DS-Ri1. Cells were imaged sequentially with DAPI (ex 350/50, em 460/50, to detect Hoechst), EGFP (ex 480/30, em $535 / 40$, to detect FDA fluorescence), TRITC (ex 540/25, em 605/ 55 , to detect PI fluorescence), with a $10 \times$ objective (NIKON Plan Fluor $10 \times / 0.30 \mathrm{WD} 16)$. For every sample, at least 5 different fields of view were acquired. FDA staining was used as a marker of cell-membrane integrity and culture viability. For each field, the ratio of PI-positive (apoptotic) nuclei over the total number of nuclei, identified by Hoechst fluorescence, was calculated. Images were analyzed by using the ImageJ software.

\section{Immunofluorescence}

The following primary antibodies were used: monoclonal and polyclonal anti-neuronal class III $\beta$-tubulin (MMS-435P, Covance, NJ and T2200, Sigma, respectively), polyclonal anti-neural cell adhesion molecule (NCAM, ab5032, Millipore, MA), monoclonal anti-phosphorylated neurofilament heavy chain (SMI-310R, Covance), monoclonal anti-pan- $\mathrm{Na}^{+}$channels (S8809, Sigma), polyclonal anti-synapsin I (ab8, Abcam, UK). Fluorescent-conjugated secondary antibodies were from Molecular Probes (Invitrogen). Cells were fixed with $4 \%$ paraformaldehyde (PFA)/20\% sucrose in phosphate buffered saline (PBS) for $15 \mathrm{~min}$ at RT and permeabilized with $0.1 \%$ TritonX100 in PBS for $5 \mathrm{~min}$ at RT. Samples were blocked for $30 \mathrm{~min}$ in IF buffer (2\% BSA, $10 \%$ goat serum in PBS). Primary and secondary antibodies were diluted in IF buffer and incubated for $45 \mathrm{~min}$ at RT. Images were acquired at an upright Leica TCS SP5 AOBS TANDEM confocal microscope equipped with a $40 \times / 0.80$ APO L W UVI objective. Images were visualized and processed by using the Leica LAS AF, ImageJ and Adobe Photoshop CS3 software.

\section{Sholl analysis}

DIV 4 neurons were fixed and stained for anti-neuronal class III $\beta$-tubulin. Images were acquired at an upright Leica TCS SP5 AOBS TANDEM confocal microscope equipped with a $20 \times / 0.50$ W UVI objective. At least 150 cells were analyzed for each condition from 3 independent cell culture preparations. Sholl analysis was performed by using the Sholl plugin of ImageJ (starting radius $10 \mu \mathrm{m}$, radius step size $10 \mu \mathrm{m}$, ending radius $200 \mu \mathrm{m})$.

\section{Scanning electron microscopy}

Neurons were grown for 7 days and then fixed for $1 \mathrm{~h}$ in a solution of $1.2 \%$ glutaraldehyde (G5882 Sigma) in $0.1 \mathrm{M}$ cacodylate buffer (pH 7.4) at $4{ }^{\circ} \mathrm{C}$. After fixation, cells were extensively washed in the same buffer, and postfixed for $1 \mathrm{~h}$ on ice in a filtered solution of $1 \%$ osmium tetroxide (O5500, Sigma) in cacodylate buffer $0.1 \mathrm{M}$. After several washes in ice-cold Milli-Q water, fixed samples were rinsed for $5 \mathrm{~min}$ in increasing concentrations of filtered ice-cold ethanol (25\%, 35\%, 50\%, $70 \%, 80 \%, 90 \%$ and $96 \%$ ), followed by $2 \times 15$ min rinses with ice-cold $100 \%$ ethanol. Graded dehydration with ethanol was followed by gradual replacement with ice-cold hexamethyldisilazane (52619, Sigma) that was allowed to evaporate in a fume hood overnight. Samples were then glued with silver paint (Pelco) to SEM stubs, coated with $10 \mathrm{~nm}$ gold/palladium in a sputter coater (108 auto/SE Cressington), and observed with a JEOL JSM-6490LA variable pressure SEM.

\section{Conclusions}

In this work, we describe a novel fabrication process to realize nanopatterned PCL films compatible with the survival and growth of primary neurons. Surface patterning mimics the structural and physical properties of the extracellular matrix, and it is fundamental to ensure optimal adhesion, survival and growth of neural networks. Such nanopatterned films are extremely promising as they are endowed with a number of attractive properties such as the ability to adhere to different substrates, and sufficient mechanical strength for sterilization and clinical handling. Furthermore, the nanofabrication procedure is fast and inexpensive, thus making our devices easily producible on large scale. Thus, we envisage our nanopatterned PCL films will be employed in a number of biomedical applications in the fields of tissue engineering and nervous system regeneration.

\section{Acknowledgements}

The authors thank Dr M. Nanni and C. Chiabrera for help with primary neuronal cultures. The research was supported by the "Single Molecule Activation and Computing" (FOCUS) European project (Grant agreement no: 270483), and by the "Rapid prototyping scaffolds for the nervous system" (neuroscaffolds) EU-China joint project (FP7-NMP-2013-EU-China).

\section{Notes and references}

1 Y. W. Fan, F. Z. Cui, S. P. Hou, Q. Y. Xu, L. N. Chen and I. S. Lee, J. Neurosci. Methods, 2002, 120, 17-23.

2 T. Limongi, F. Cesca, F. Gentile, R. Marotta, R. Ruffilli, A. Barberis, M. Dal Maschio, E. M. Petrini, S. Santoriello, F. Benfenati and E. Di Fabrizio, Small, 2013, 9, 402-412.

3 S. L. Gupton and C. M. Waterman-Storer, Cell, 2006, 125, 1361-1374.

4 M. J. Biggs, R. G. Richards and M. J. Dalby, Nanomedicine: Nanotechnology, Biology and Medicine, 2010, 6, 619-633. 
5 J. P. Myers, M. Santiago-Medina and T. M. Gomez, Dev. Neurobiol., 2011, 71, 901-923.

6 N. J. Gardiner, Dev. Neurobiol., 2011, 71, 1054-1072.

7 J. J. Norman and T. A. Desai, Ann. Biomed. Eng., 2006, 34, 89101.

8 Q. P. Pham, U. Sharma and A. G. Mikos, Tissue Eng., 2006, 12, 1197-1211.

9 A. Nandakumar, Z. Tahmasebi Birgani, D. Santos, A. Mentink, N. Auffermann, K. van der Werf, M. Bennink, L. Moroni, C. van Blitterswijk and P. Habibovic, Biofabrication, 2013, 5, 015006.

10 T. Jacobs, R. Morent, N. De Geyter, P. Dubruel and C. Leys, Plasma Chem. Plasma Process., 2012, 32, 1039-1073.

11 Q. Cheng, B. L. Lee, K. Komvopoulos, Z. Yan and S. Li, Tissue Eng., Part A, 2013, 19, 1188-1198.

12 U. A. Sezer, E. A. Aksoy, V. Hasirci and N. Hasirci, J. Appl. Polym. Sci., 2013, 127, 2132-2139.

13 M. Rampichova, J. Chvojka, M. Buzgo, E. Prosecka, P. Mikes, L. Vyslouzilova, D. Tvrdik, P. Kochova, T. Gregor, D. Lukas and E. Amler, Cell Proliferation, 2013, 46, 23-37.
14 J. Korpela, A. Kokkari, H. Korhonen, M. Malin, T. Narhi and J. Seppala, J. Biomed. Mater. Res., Part B, 2013, 101, 610-619.

15 L. Ghasemi-Mobarakeh, M. P. Prabhakaran, M. Morshed, M. H. Nasr-Esfahani and S. Ramakrishna, Mater. Sci. Eng., C, 2010, 30, 1129-1136.

16 P. S. Donoghue, R. Lamond, S. D. Boomkamp, T. Sun, N. Gadegaard, M. O. Riehle and S. C. Barnett, Tissue Eng., Part A, 2013, 19, 497-507.

17 F. Cesca, A. Yabe, B. Spencer-Dene, J. Scholz-Starke, L. Medrihan, C. H. Maden, H. Gerhardt, I. R. Orriss, P. Baldelli, M. Al-Qatari, M. Koltzenburg, R. H. Adams, F. Benfenati and G. Schiavo, Cell Death Differ., 2012, 19, 194-208.

18 A. Cooper, N. Bhattarai and M. Zhang, Carbohydr. Polym., 2011, 85, 149-156.

19 S. De Faveri, E. Maggiolini, E. Miele, F. De Angelis, F. Cesca, F. Benfenati and L. Fadiga, Front. Neuroeng., 2014, 7, 7. 